\title{
Sufficient conditions for symmetric matrices to have exactly one positive eigenvalue
}

https://doi.org/10.1515/spma-2020-0009

Received October 24, 2019; accepted March 18, 2020

Abstract: Let $A=\left[a_{i j}\right]$ be a real symmetric matrix. If $f:(0, \infty) \longrightarrow[0, \infty)$ is a Bernstein function, a sufficient condition for the matrix $\left[f\left(a_{i j}\right)\right]$ to have only one positive eigenvalue is presented. By using this result, new results for a symmetric matrix with exactly one positive eigenvalue, e.g., properties of its Hadamard powers, are derived.

Keywords: Bernstein function, Hadamard power, Hadamard inverse, distance matrix, infinitely divisible matrix, conditionally negative semidefinite matrix.

MSC: 15B48, 15B57, 15A18, 26A48.

\section{Introduction}

A function $f:(0, \infty) \longrightarrow \mathbb{R}$ is said to be completely monotonic, if $f$ has derivatives of all orders and satisfies the inequality

$$
(-1)^{n} f^{(n)}(x) \geq 0, x>0 \text { and } n=0,1,2, \ldots
$$

A differentiable function $f:(0, \infty) \longrightarrow[0, \infty)$ is called a Bernstein function, if $f^{\prime}$ is completely monotonic. For example, the elementary function $f(x)=x^{\alpha}, 0<\alpha \leq 1$, is a Bernstein function.

The Bernstein functions on $(0, \infty)$ can be characterized as follows:

A function $f:(0, \infty) \longrightarrow[0, \infty)$ is a Bernstein function if and only if it admits the representation

$$
f(x)=\alpha+\beta x+\int_{0}^{\infty}\left(1-e^{-t x}\right) d \mu(t),
$$

where $\alpha, \beta \geq 0$ and $\mu$ is a measure on $(0, \infty)$ satisfying $\int_{0}^{\infty} \min \{1, t\} d \mu(t)<\infty$, see, e.g., [12, Theorem 3.2].

The notion of the Bernstein function goes back to the treatment of potential theory in the work of the school of J. Deny, which was the source of inspiration for [15]. In [5], a Bernstein function is called a completely monotone function, which was characterized by Bernstein in 1929 as a Laplace transform of positive measure on $[0, \infty)$, see [4]. The literature devoted to this class of functions is impressive since they have remarkable applications in various branches, for instance, they play a role in potential theory, probability theory, physics, numerical and asymptotic analysis, and combinatorics. A detailed collection of the most important properties as well as lists of Bernstein functions can be found in the survey paper [3] and the monograph [12].

Let $A=\left[a_{i j}\right]$ and $B=\left[b_{i j}\right]$ be real $n \times n$ matrices. Their Hadamard product (also called Schur product) $A \circ B$ is defined as the entrywise product of $A$ and $B, A \circ B=\left[a_{i j} b_{i j}\right]$. The Hadamard unit matrix is the matrix $E$ all of

Doaa Al-Saafin: Department of Mathematics and Statistics, University of Konstanz, Germany,

E-mail: doaa-mahmoud.al-saafin@uni-konstanz.de

*Corresponding Author: Jürgen Garloff: Department of Mathematics and Statistics, University of Konstanz, and Institute for Applied Research, University of Applied Science / HTWG Konstanz, Germany, E-mail: juergen.garloff@htwg-konstanz.de 
whose entries are 1 (the size of $E$ being understood). A matrix is Hadamard invertible if all its entries are nonzero, and $A^{\circ-1}=\left[1 / a_{i j}\right]$ is then called the Hadamard inverse of $A$. If all entries of $A$ are non-negative, then the $r$-th Hadamard power of $A$ is $A^{\circ r}=\left[a_{i j}^{r}\right], r>0$. We define the Hadamard exponential of $A$ by $e^{\circ A}=\left[e^{a_{i j}}\right]$ and, if $A$ has all entries positive, the Hadamard logarithm of $A$ by $\log ^{\circ}(A)=\left[\log \left(a_{i j}\right)\right]$. Suppose that $A$ is positive semidefinite and that $a_{i j} \geq 0$ for all $i$ and $j$. We say that $A$ is infinitely divisible if the matrix $A^{\text {or }}$ is positive semidefinite for every non-negative $r$. If $A$ is a symmetric matrix such that $a_{i j}>0$ for all $i, j$ and $A$ has exactly one positive eigenvalue, then we say that $A$ is in the class $\mathcal{A}$.

In this paper, we present a result for the Hadamard power of matrices in class $\mathcal{A}$. From this result follows an alternative proof of a result due to Bapat [1, p.471, proof of Lemma 6], who showed that if $A$ is in class $\mathcal{A}$, then its Hadamard inverse is infinitely divisible.

Let $\mathbf{e} \in \mathbb{R}^{n}$ be the vector of all ones and $\mathbf{e}_{i} \in \mathbb{R}^{n}$ be the vector which has in its $i$-th component a one, while all its other components are zero. A real symmetric $n \times n$ matrix $A$ is said to be conditionally positive (negative) semidefinite if $\mathbf{x}^{T} A \mathbf{x} \geq 0$ ( $\leq 0$ ) for all $\mathbf{x} \in \mathbb{R}^{n}$ such that $\mathbf{x}^{T} \mathbf{e}=0 ; A$ is conditionally positive (negative) definite if $\mathbf{x}^{T} A \mathbf{x}>0(<0)$ for all $\mathbf{x} \in \mathbb{R}^{n}$ such that $\mathbf{x}^{T} \mathbf{e}=0$.

It is well-known that a non-zero conditionally negative semidefinite matrix with all entries non-negative has exactly one positive eigenvalue, see [2, Corollary 4.1.5]. By using a result on Bernstein functions, we present new results for conditionally negative semidefinite matrices with positive entries.

In [2, Theorem 4.4.6], Bapat and Raghavan proved a sufficient and necessary condition based on the signed principal minors for a symmetric matrix with positive entries to have exactly one positive eigenvalue. We prove a similar sufficient condition. A consequence of this result will be used to show that if $A$ is a symmetric matrix which has all off-diagonal entries positive, all diagonal entries zero, and has just one positive eigenvalue, then $A^{\circ \alpha}, 0<\alpha<1$, has one positive eigenvalue. This result provides a generalization of a theorem given by Reams [11]. We will use the Perron-Frobenius Theorem, which states that if an irreducible matrix $A$ has all its entries nonnegative then it has a positive eigenvalue $\mu$. Furthermore, the eigenvector that corresponds to $\mu$, called the Perron eigenvector of $A$, can be chosen as a vector with positive components., see, e.g., [8, Theorem 8.4.4].

\section{Background and key lemmata}

We collect here some key facts needed for our main results. The following lemmata are well-known.

Lemma 1. (Schur Product Theorem): Suppose $A$ and $B$ are positive semidefinite matrices of size $n$. Then $A \circ B$ is also positive semidefinite. If $A$ and $B$ are positive definite, then $A \circ B$ is positive definite, too.

Lemma 2. ([7, p.144]; [9, Corollary 2.1]):

The symmetric matrix $A=\left[a_{i j}\right] \in \mathbb{R}^{n \times n}$ is conditionally positive semidefinite if and only if its Hadamard exponential $e^{\circ t A}$ is positive semidefinite for all $t \geq 0$. Moreover, $e^{\circ t A}$ is positive definite for all $t>0$ if and only if $2 a_{i j}<a_{i i}+a_{j j}$, for all $i \neq j$.

Lemma 3. ([2, Corollary 4.4.5, Theorem 4.4.6]; [11, Corollary 2.8, proof of Theorem 2.7]): Let $A=\left[a_{i j}\right] \in \mathcal{A}$. Then the following properties hold:

(i) The Hadamard inverse of $A$ is positive semidefinite. Moreover, it is positive definite if $A$ is invertible.

(ii) If $A$ is conditionally negative definite, then the Hadamard inverse of $A$ is positive definite if and only if $2 a_{i j}>a_{i i}+a_{j j}$, for all $i \neq j$.

(iii) The matrix $A \circ\left(\mathbf{v v}^{T}\right)^{\circ-1}$ is conditionally negative semidefinite, where $\mathbf{v}$ is the Perron eigenvector of $A$.

Lemma 4. ([2, Lemma 4.3.5]):

Let $A$ be a symmetric conditionally negative semidefinite matrix that is not negative semidefinite, and suppose that $\mathbf{x}^{T} A \mathbf{x} \neq 0$ for every non-zero $\mathbf{x} \in \mathbb{R}^{n}$ such that $\mathbf{x}^{T} \mathbf{e}=0$, then $A$ is invertible. 
Lemma 5. (Sylvester's Law of Inertia, e.g., [8, Theorem 4.5.8]):

Let $A$ and $B$ be Hermitian matrices, then there exists a non-singular matrix $S$ such that $A=S B S^{\star}$ if and only if $A$ and $B$ have the same inertia.

\section{Main results}

Theorem 6. Let $A=\left[a_{i j}\right] \in \mathbb{R}^{n \times n}$ be a symmetric conditionally negative semidefinite matrix with all entries positive and let $f:(0, \infty) \longrightarrow[0, \infty)$ be a Bernstein function. Then $\left[f\left(a_{i j}\right)\right] \in \mathcal{A}$. Moreover, it is invertible if $A$ is invertible.

Proof. Let $f$ be a Bernstein function, thus by (1),

$$
f\left(a_{i j}\right)=\alpha+\beta a_{i j}+\int_{0}^{\infty}\left(1-e^{-t a_{i j}}\right) d \mu(t),
$$

where $\alpha, \beta \geq 0$ and $\left[\alpha+\beta a_{i j}\right]$ is conditionally negative semidefinite.

Since $A$ is conditionally negative semidefinite, $-t A$ is conditionally positive semidefinite and so by Lemma $2,\left[e^{\circ-t A}\right]$ is positive semidefinite for all $t>0$. Therefore, $\left[E-e^{\circ-t A}\right]$ is conditionally negative semidefinite. Whence $\left[f\left(a_{i j}\right)\right]$ is conditionally negative semidefinite, and hence it has one positive eigenvalue.

To prove the sufficient condition on the invertibility of $\left[f\left(a_{i j}\right)\right]$, suppose $A$ is in addition invertible. Then, Lemma 3(i), (ii) implies that $2 a_{i j}>a_{i i}+a_{j j}$, for all $i \neq j$ and so $-A$ is invertible, conditionally positive semidefinite and has the property $-2 a_{i j}<-a_{i i}-a_{j j}$, for all $i \neq j$. By Lemma 2, [ $\left.e^{\circ-t A}\right]$ is positive definite and hence $\left[-e^{\circ-t A}\right]$ is conditionally negative definite for all $t>0$. This implies that $\left[E-e^{\circ-t A}\right]$ is conditionally negative definite. Therefore, $\left[f\left(a_{i j}\right)\right]$ is conditionally negative definite and since it has a positive eigenvalue, it is not negative semidefinite. By using Lemma 4, we conclude that $\left[f\left(a_{i j}\right)\right]$ is invertible.

Theorem 7. Let $A \in \mathcal{A}$. Then $A^{\circ \alpha}$ belongs also to $\mathcal{A}$, where $0<\alpha \leq 1$ and is invertible if $A$ is invertible.

Proof. Let $A \in \mathcal{A}$ and let $\mathbf{v}$ be the Perron eigenvector of $A$. By Lemma 3(iii), $B:=A \circ\left(\mathbf{v v}^{T}\right)^{\circ-1}$ is conditionally negative semidefinite and hence it has exactly one positive eigenvalue. For $0<\alpha \leq 1$, the function $f(x)=x^{\alpha}$ is a Bernstein function. Thus, by Theorem $6, B^{\circ \alpha}$ has exactly one positive eigenvalue and is invertible if $B$ is invertible, where $0<\alpha \leq 1$. Let $W=\operatorname{diag}\left(v_{1}^{\alpha}, \ldots, v_{n}^{\alpha}\right)$, then $A^{\circ \alpha}=W B^{\circ \alpha} W$. By Lemma $5, A^{\circ \alpha}$ has one positive eigenvalue and is invertible if $B^{\circ \alpha}$ is invertible. Finally, by Lemma 5, $B$ is invertible if $A$ is invertible.

In Corollary 8, we give an alternative proof of a result due to Bapat [1, p.417, proof of Lemma 6] and provide some results for the conditionally negative semidefinite matrices.

Corollary 8. Let $A \in \mathcal{A}$, then the Hadamard inverse of $A$ is infinitely divisible.

Proof. Let $A \in \mathcal{A}$, then the proof of the positive definiteness of $A^{\circ-\alpha}$ for all $0<\alpha \leq 1$ follows directly from Theorem 7 and Lemma 3(i). The extension to all $\alpha>1$ is provided by the application of Lemma 1.

The application of Theorem 6 and Corollary 8 to Bernstein functions can be used to obtain the infinite divisibility of some matrices. The following theorem presents three examples.

Theorem 9. Let $A$ be a symmetric conditionally negative semidefinite matrix with positive entries, then the following matrices are infinitely divisible:

(i) $E+r A^{\circ-1}, r>0$,

(ii) $\left(\log ^{\circ}(E+A)\right)^{\circ-1}$,

(iii) $(A+\alpha E)^{\circ-1} \circ(A+\beta E), 0 \leq \alpha<\beta$. 
Proof. Assume that $x \in(0, \infty)$. (i): The function $f(x)=\frac{x}{r+x}$, where $r>0$, is a Bernstein function. Thus, if $A$ is conditionally negative semidefinite with positive entries, Theorem 6 and Corollary 8 imply (i).

(ii): The proof follows by using the same arguments as in the proof of (i) for the Bernstein function $g(x)=$ $\log (1+x)$.

(iii): By [15, p.9], the following representation holds

$$
\log \frac{x+\beta}{x+\alpha}=\int_{\alpha}^{\beta}(x+\xi)^{-1} d \xi,
$$

where $0 \leq \alpha<\beta$ and $\xi$ is a measure on $(0, \infty)$. Now, if a symmetric matrix $A$ is conditionally negative semidefinite with positive entries, then (2) implies that the matrix $\left[\log \frac{a_{i j}+\beta}{a_{i j}+\alpha}\right]$ is positive semidefinite. We complete the proof by using Lemma 2 .

Theorem 10. Let $A \in \mathcal{A}$, then for all $r>0, r A+\mathbf{v v}^{T} \in \mathcal{A}$, where $\mathbf{v}$ is the Perron eigenvector of $A$.

Proof. Let $A=\left[a_{i j}\right] \in \mathcal{A}$, then Lemma 3(ii) ensures that the matrix $\left[r \frac{a_{i j}}{v_{i} v_{j}}+1\right]$ is in class $\mathcal{A}$. We complete the proof in the same way as in the proof of Theorem 7.

The next two lemmata will be used in the proof of Theorem 13 . Note that, if a symmetric matrix $A=\left[a_{i j}\right] \in \mathbb{R}^{n \times n}$ is conditionally negative definite, then by $\left(\mathbf{e}_{i}-\mathbf{e}_{j}\right)^{T} \mathbf{e}=0$, we have $0>\left(\mathbf{e}_{i}-\mathbf{e}_{j}\right)^{T} A\left(\mathbf{e}_{i}-\mathbf{e}_{j}\right)=a_{i i}+a_{j j}-2 a_{i j}$.

It was shown in [2, Theorem 4.4.6] and follows from [10, Theorem 3.1] by Lemma 5, that a symmetric matrix $A \in \mathbb{R}^{n \times n}$ with positive entries belongs to the class $\mathcal{A}$ if and only if, for any $k \times k$ principal submatrix $B$ of $A,(-1)^{k-1} \operatorname{det} B \geq 0$, for all $k=1, \ldots, n$. The next lemma provides a weaker condition for a symmetric matrix to have exactly one positive eigenvalue by removing the assumption of the positivity of the entries of $A$.

Lemma 11. ([14, Lemma 3.8]):

Let $A \in \mathbb{R}^{n \times n}$ be a symmetric matrix and let for each $k \times k$ leading principal submatrix $B$ of $A,(-1)^{k-1} \operatorname{det} B>0$, for $k=1, \ldots, n$, then $A$ has exactly one positive eigenvalue.

The following lemma was proven in [9] for Euclidean distance matrices, where in Remark 3.2 therein it is mentioned that the proof can be extended to arbitrary symmetric conditionally negative semidefinite matrices with nonnegative entries satisfying the inequality condition in Lemma 2, cf. [6, pp.163-164].

Lemma 12. ([9]):

If a symmetric matrix $A=\left[a_{i j}\right] \in \mathbb{R}^{n \times n}$ is conditionally negative semidefinite with nonnegative entries and

$$
2 a_{i j}>a_{i i}+a_{j j} \text {, for all } i \neq j,
$$

then

$$
(-1)^{n-1} \operatorname{det}\left(A^{\circ \alpha}\right)>0
$$

for all $0<\alpha<1$.

A famous theorem of Schoenberg in distance geometry says that a symmetric matrix $D=\left[d_{i j}\right]$ with zero diagonal entries and positive off-diagonal entries, is conditionally negative semidefinite if and only if there exist distinct vectors $\mathbf{u}_{1}, \ldots, \mathbf{u}_{n} \in \mathbb{R}^{s}$ for some $s$ such that $\left\|\mathbf{u}_{i}-\mathbf{u}_{j}\right\|^{2}=d_{i j}$ for all $i, j$, i.e., $D$ is an Euclidean distance matrix, see [2, Theorem 4.1.7]. In [9, proof of Theorem 2.3], Micchelli showed that if a conditionally negative semidefinite matrix $A \in \mathbb{R}^{n \times n}$ is a distance matrix, then $A^{\circ \alpha}, 0<\alpha<1$, has one positive eigenvalue and is invertible. In the next theorem, we give a weaker condition on $A$ such that $A^{\circ \alpha}$ has one positive eigenvalue.

Theorem 13. Let $A \in \mathbb{R}^{n \times n}$ be a symmetric conditionally negative semidefinite matrix with nonnegative entries and

$$
2 a_{i j}>a_{i i}+a_{j j} \text {, for all } i \neq j \text {. }
$$


Then $A^{\circ \alpha}, 0<\alpha<1$, has one positive eigenvalue and is invertible.

Proof. From the hypothesis, we can conclude that $-A$ is conditionally positive semidefinite and $-2 a_{i j}<-a_{i i}-$ $a_{j j}$, for all $i \neq j$. Let $B$ be any leading principal submatrix of $A$. By Lemma 2, $e^{\circ-A}$ is positive definite. Then $e^{\circ-B}$ is also positive definite. By Lemma 2 we conclude that $B$ is conditionally negative definite and has the property $2 b_{i j}>b_{i i}+b_{j j}$, for all $i \neq j$. Now, Lemma 12 and Lemma 11 imply that the matrix $A^{\circ \alpha}$, has one positive eigenvalue for all $0<\alpha<1$ and is invertible.

In [11, Theorem 2.9], Reams showed that if a symmetric matrix $A$, with positive off-diagonal entries and all diagonal entries are zero, has one positive eigenvalue, then the Hadamard square root of $A$ has also one positive eigenvalue and is invertible. The next theorem gives a generalization of his result which was already proven by Schoenberg in [13] for the special case of distance matrices (of distinct points).

Theorem 14. Let $A \in \mathbb{R}^{n \times n}$ be symmetric with positive off-diagonal entries, zero diagonal entries, and just one positive eigenvalue. Then $A^{\circ \alpha}, 0<\alpha<1$, has one positive eigenvalue and is invertible.

Proof. Let $\lambda_{1} \leq \cdots \leq \lambda_{n-1} \leq 0<\lambda_{n}$ be the eigenvalues of $A$ with $A \mathbf{v}=\lambda_{n} \mathbf{v}$ and $A \mathbf{u}_{j}=\lambda_{j} \mathbf{u}_{j}$, where $1 \leq j \leq n-1$ and $\mathbf{v}=\left(v_{1}, \ldots, v_{n}\right)^{T} \in \mathbb{R}^{n}$ be the Perron eigenvector of $A$.

Write

$$
A=\lambda_{n} \mathbf{v} \mathbf{v}^{T}+\lambda_{n-1} \mathbf{u}_{n-1} \mathbf{u}_{n-1}^{T}+\cdots+\lambda_{1} \mathbf{u}_{1} \mathbf{u}_{1}^{T} .
$$

Then,

$$
\mathbf{x}^{T} W A W \mathbf{x}=\mathbf{x}^{T}\left(\lambda_{n} \mathbf{e}^{T}+\lambda_{n-1}\left(W \mathbf{u}_{n-1}\right)\left(W \mathbf{u}_{n-1}\right)^{T}+\cdots+\lambda_{1}\left(W \mathbf{u}_{1}\right)\left(W \mathbf{u}_{1}\right)^{T}\right) \mathbf{x},
$$

where $W=\operatorname{diag}\left(\frac{1}{v_{1}}, \ldots, \frac{1}{v_{n}}\right)$. So, if $\mathbf{x}^{T} \mathbf{e}=0$, then

$$
\mathbf{x}^{T} W A W \mathbf{x} \leq 0,
$$

and hence the matrix $W A W$ is conditionally negative semidefinite. By using Theorem $13,(W A W)^{\circ \alpha}=$ $W^{\circ \alpha} A^{\circ \alpha} W^{\circ \alpha}$ has one positive eigenvalue. If $A^{\circ \alpha}$ would not be invertible then $W^{\circ \alpha} A^{\circ \alpha} W^{\circ \alpha}$ would not be invertible by Lemma 5 and we obtain by (3) a contradiction to the positivity of the off-diagonal entries of $A$.

\section{Conclusion}

In our paper, we have extended some known results related to the class of symmetric matrices having exactly one positive eigenvalue, e.g., on their Hadamard powers and inverses.

Note added in proof: When our paper was already in proof we learnt that a statement similar to Theorem 6 for operator monotone functions was given in I. Garg, J. S. Aujla, Inertia of non-integer Hadamard powers of a non-negative matrix. Linear Multilinear Algebra, 68(2):410-416, 2020.

\section{References}

[1] R. B. Bapat, Multinomial probabilities, permanents and a conjecture of Karlin and Rinott. Proc. Amer. Math. Soc., 102(3):467472, 1988.

[2] R. B. Bapat, T. E. S. Raghavan, Nonnegative Matrices and Applications. Encyclopedia Math. Soi. Vol. 64. Cambridge University Press, Cambridge, UK, 1997.

[3] C. Berg, Stieltjes-Pick-Bernstein-Schoenberg and their connection to complete monotonicity, In: Positive Definite Functions: from Schoenberg to Space-Time Challenges, S. Mateu and E.Porcu (eds.), Dept. Math., Univ. Jaume I, Castellón de la Plana, Spain, 2008, 24 pages; Available online at http://citeseerx.ist.psu.edu/viewdoc/summary?doi=10.1.1.142.3872.

[4] S. Bernstein, Sur les fonctions absolument monotones. Acta Math, 52:1-66, 1929.

[5] S. Bochner, Harmonic Analysis and the Theory of Probability. University of California Press, Berkeley and Los Angeles, 1955. 
[6] N. Dyn, T. Goodman, C. A. Micchelli, Positive powers of certain conditionally negative delinite matrices. Indag. Math. (Proceedings), 89(2): 163-178, 1986.

[7] R. A. Horn, The Hadamard product. Proc. Sympos. Appl. Math, 40: 87-169, 1990.

[8] R. A. Horn, C. R. Johnson, Matrix Analysis, 2nd ed. Cambridge University, New York, 2013.

[9] C. A. Micchelli, Interpolation of scattered data: distance matrices and conditionally positive definite functions. Constr. Approx., 2:11-22, 1986.

[10] J. M. Peña, Positive symmetric matrices with exactly one positive eigenvalue. Linear Algebra Appl., 430:1566-1573, 2009.

[11] R. Reams, Hadamard inverses, square roots and products of almost semidefinite matrices. Linear Algebra Appl., 288:35-43, 1999.

[12] R. L. Schilling, R. Song, Z. Vondraček, Bernstein Functions: Theory and Applications, 2nd ed. de Gruyter Stud. Math. Vol. 37. de Gruyter, Berlin, 2012.

[13] I. J. Schoenberg, On certain metric spaces arising from euclidean spaces by a change of metric and their imbedding in Hilbert space. Ann. of Math. (2), 38(4):787-793, 1937.

[14] S. Q. Shen, T. Z. Huang, On symmetric matrices with exactly one positive eigenvalue. Electron. J. Linear Algebra, 20:158-167, 2010.

[15] C. Van Den Berg, G. Forst, Potential Theory on Locally Compact Abelian Groups. Ergebnisse der Mathematik und ihrer Grenzgebiete, Band 87. Springer-Verlag, Berlin, Heidelberg, New York, 1975. 